\title{
Computed tomography soft tissue restoration using Wiener filter
}

\begin{abstract}
This paper presents image restoration for Computed Tomography (CT) scan image particularly soft tissue area using image restoration technique. The approach of these technique are using inverse filtering which specifically involving Wiener filter to remove the noise and blur simultaneously in the CT image further restore the degraded CT image. CT scan image usually showing less detail visualization of soft tissue compare to Magnetic Resonance Imaging (MRI) scan image. The new CT image after restoration technique showed significantly high contrast compared to the original CT image produced by the CT scan machine. This procedure also shows comparison of different values use for noise to signal ratio (NSR) in Wiener filter equation to generate the estimate output of CT image. In this procedure values of NSR are treat as constant.
\end{abstract}

Keyword: Computed tomography; Magnetic resonance imaging; Soft tissue; Wiener filter 